Original Article

\title{
Assessment of physical health status and quality of life among Saudi older adults
}

\author{
Samira Al Senany ${ }^{1 *}$, Amer Al Saif ${ }^{2)}$ \\ 1) Department of Public Health, Faculty of Nursing, King Abdulaziz University: Jeddah, Saudi Arabia \\ 2) Department of Physical Therapy, Faculty of Applied Medical Sciences, King Abdulaziz University, \\ Saudi Arabia
}

\begin{abstract}
Purpose] This study investigated physical health status and quality of life among older Saudi adults. [Subjects and Methods] Participants included older adults $(\mathrm{N}=55)$ aged 60-90 years (mean $=67.9 \pm 7.71)$ from a major hospital in Jeddah. Subjects completed surveys and evaluations including assessments of personal and sociodemographic characteristics, caregiver presence, personal habits, perceived health, primary physical and psychological complaints, physician-diagnosed chronic diseases, and functional capacity (i.e., activities of daily living). [Results] Results showed a significant positive correlation between age and ADL; age and memory problems, anxiety, and loneliness; and sleep disturbance and falls. Main factors contributing to quality of life decline were chronic disease, falls, sedentary lifestyle, sleep disturbances, and financial concerns. Participants with diabetes mellitus $(58.18 \%)$ and hypertension $(29.0 \%)$ had a very high fall rate. Participants engaged in minimal physical activity $(63 \%)$, often due to bone and joint pain $(90.0 \%)$, and led sedentary lives $(69 \%)$. Single sensory impairments were significantly and independently linked to high depression risk and decreased self-sufficiency. [Conclusion] Healthy lifestyle and behavioral changes should be promoted through community-based health education programs for older Saudi adults. Chronic disease screening programs for the elderly population (especially diabetes and hypertension) are recommended.

Key words: Elderly, Falls, Depression
\end{abstract}

(This article was submitted Jan. 5, 2015, and was accepted Feb. 14, 2015)

\section{INTRODUCTION}

People over age 60 numbered around 600 million worldwide in 2000, and these figures are expected to reach 1.2 billion by 2025 and 2 billion by 2050 . Of these, about two thirds currently live in the developing world, and it is estimated that this figure will rise to $75 \%$ by $2025^{1)}$. Globally, this increase in both overall numbers of older people and their percentage relative to the total population has considerable implications for older people and service availability $^{2)}$. Specifically, this will lead to changing demands on health care systems in both developing and developed countries and Saudi Arabia is no exception. The World Health Organization (WHO) defines quality of life (QoL) as an individual's perception of their position in life in the context of their culture and value systems and in relation to their goals, expectations, standards and, concerns. It is a broad concept complexly affected by the person's physical health, psychological state, personal beliefs, social relationships, and relationship with environmental features ${ }^{1)}$. The

*Corresponding author. Samira Al Senany (E-mail: salsenany@kau.edu.sa)

C2015 The Society of Physical Therapy Science. Published by IPEC Inc. This is an open-access article distributed under the terms of the Creative Commons Attribution Non-Commercial No Derivatives (by-ncnd) License $<$ http://creativecommons.org/licenses/by-nc-nd/3.0/>.
Kingdom of Saudi Arabia is one of the largest countries in the Middle East, and during recent decades has experienced a rapid expansion in health services. The health care system in Saudi Arabia can be divided into government and private sectors, with free-of-charge government services for Saudi and non-Saudi individuals, with very few restrictions. Saudi Arabia provides primary, secondary, and tertiary health care services. There are large, technologically advanced hospitals in major cities that provide open-heart surgery, kidney transplantation, and oncology therapy. However, despite expansion, health expenditures are expected to dramatically increase and exceed the population growth rate as hospital bed demand grows. Several key issues will influence health care delivery in this society. While the proportion of the elderly population to total population was $5.8 \%$ in 2000 , it is expected to reach $8.7 \%$ by 2026 and $15.0 \%$ by 2050 . This demographic transition of population aging is a global phenomenon that demands international, national, regional, and local action. Consequently, there has been increasing international awareness of health issues relating to aging populations. Consequently, Saudi adults are becoming older and the percentage of the population over 60 is rising, and is expected to more than double by 2020 . By this date, the number of older people is expected to grow from approximately 1 million ( $4 \%$ of the population) to roughly 2.5 million $(7 \% \text { of the population })^{1,2)}$. This increased number of older people in Saudi Arabia presents numerous challenges to health care system, and especially to health care work- 
ers providing services to older clients with both acute and chronic conditions. The current changes to the health care system and shortage of health care personnel have made it difficult for older adults to access adequate health care of suitable quality. In light of the above, health care provision for older people in Saudi Arabia is not yet as well developed as general services, and studies concerning older peoples' needs are scarce. The Saudi cultural tradition for supporting older people puts a premium on personal family care, especially for those that are partially disabled, sick, and older, remains strong in the Kingdom, and is largely underpinned by religious faith. In general, old age is admired as a holy state of great religious significance and the aged are given considerable respect within the family in Eastern cultures.

In the home environment, an older person's views usually prevail. Thus, older patients would not expect any dramatic changes to the way they are accustomed to being treated in health care facilities. However, the family emphasis potentially raises tensions with formal support. Whilst there is no doubt that the older population is growing and increasing older people's adequate health care needs, culture dictates that the family is primarily responsible for care. Such cultural and religious considerations pose unique challenges to service providers when entering homes, particularly of older persons unaccustomed to the professions of social workers or in-home nurses. Furthermore, it is regarded as religiously condemnable and socially irresponsible for children to send their parents to nursing homes rather than providing home care $^{3)}$. However, the severity of older peoples' needs has increased, and nursing home health care is expected to significantly grow in the coming years in Saudi Arabia ${ }^{4}$, which is a primary concern for health care providers. Moreover, older patients are currently dissatisfied with health services in Saudi Arabia as the result of a lack of specialty clinics and skilled nursing ${ }^{5)}$. There is also a limited response to older people's care in acute care hospitals in Saudi Arabia. Similarly, one study claims that hospitals are not ideal for older persons with long-term conditions who have high accident rates $(62.5 \%)$ that primarily occur in patients aged 60 years or older ${ }^{6}$. It is suggested that may be the result, in part, to nursing staff lacking the time and skills necessary to provide longer-term hospital care for older patients ${ }^{7}$. Clearly, there is a need not only for more adult care training in Saudi Arabia, but for such training to equip health care providers with the skills needed to support older people. Any attempt to provide high quality care for older people must consider both attitudes towards these patients and knowledge of the ageing process.

\section{SUBJECTS AND METHODS}

A cross-sectional study was conducted among elderly individuals (aged 60 years and older) in a major hospital in Jeddah, Saudi Arabia. Participants included 55 male and female

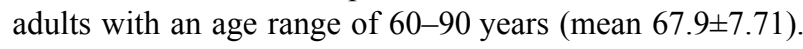
This study was approved by the Institutional Review Board at King Abdulaziz University. Prior to beginning the study, informed consent was acquired. Potential participants were recruited based on the following criteria: (1) aged 60 and older, and (2) ability to participate in activities of daily living
(ADL) independently with or without using an ambulatory aid. Data were collected using an interview questionnaire, records review, and by taking some measurements. As recommended by previous studies, QoL is best assessed using direct patient interviews. During assessment, researchers were careful not to reveal their own biases. Determining a patient's preferences is usually possible with this method and even patients with mild dementia or cognitive impairment can report preferences when researchers use simple explanations and questions. In this study, it was recommended that family members were present when discussing patient preferences for those with a cognitive impairment.

Subjects were instructed that they had the right to withdraw from the study at any time. All subjects were asked to complete the questionnaire. The questionnaire provided important background information regarding general health, which consists of each participant's ADLs, fall risk, health status (Diabetic mellitus (DM), Hypertension (HTN), heart disease, kidney, rheumatoid arthritis, osteoporosis), memory problems, sleep patterns, economic status, psychological status, educational level, and caregiver presence. This information allowed us to establish subjects' health status and determine if they had any problems that could significantly affect their QoL. Additionally, a pre-designed questionnaire was used to collect information about personal and sociodemographic characteristics, caregiver presence, personal habits, and perceived health. Subjects' functional capacity was established by asking about their ability to complete basic activities, such as walking, bathing, using the toilet, dressing, eating, and getting in and out of the home without needing help. Enquiries were made about their main physical and psychological complaints. In addition, the presence of chronic diseases was identified by asking about physiciandiagnosed chronic disease. Subjects' weight and height were measured, and body mass index (BMI) was calculated $(\mathrm{BMI}=$ weight in $\mathrm{kg} /$ height in meters squared). Data were analyzed using the statistical package SPSS for Windows Version 16.0 (SPSS, Inc., Chicago, IL, USA). The level of statistical significance was set at $\mathrm{p}<0.5$.

\section{RESULTS}

The results showed a significant positive correlation between age and ADL; age and memory problems, anxiety, and loneliness; and sleep disturbance and falls. This study also found that the main factors contributing to QoL among older adults were chronic disease, falls, sedentary lifestyle, sleep disturbance, and financial situation. Furthermore, there was an alarmingly high rate of falls among older adults with DM (58.18\%) and HTN (29.0\%). In addition, this study confirmed that older Saudi adults engaged in little physical activity (63\%) due to bone and joint pain $(90.0 \%)$ and led a sedentary life $(69 \%)$. These factors can predispose them to risk of developing a disability and experiencing falls. Furthermore, this study indicated that single sensory impairments (either visual or auditory) are significantly and independently linked with a high risk of depression and decreased self-sufficiency in daily living activities, which can adversely affect mobility and the ability to perform ADL (Tables 1-5). 
Table 1. Functional characteristics, Mean and percentages among older Saudi adults

\begin{tabular}{lc}
\hline Mean age (years) & $67.9 \pm 7.71$ \\
\hline Education (years) & $10.7 \pm 3.7$ \\
Marital status (married) & $(92.9 \%)$ \\
Vision problem & $(82.0 \%)$ \\
Hearing problem & $(54.54 \%)$ \\
Mild-moderate handicap & $(16.3 \%)$ \\
Severe handicap & $(11 \%)$ \\
Mental & $(32.1 \%)$ \\
Diabetes & $(58.18 \%)$ \\
Cancer & $(27.2 \%)$ \\
Stroke & $(21.8 \%)$ \\
Hypertension & $(29.0 \%)$ \\
Coronary & $(69.9 \%)$ \\
Bones/joints & $(90.9 \%)$ \\
Back/neck & $(70.9 \%)$ \\
Brain/neuro & $(69.0 \%)$ \\
Tobacco use & $(32.1 \%)$ \\
\hline
\end{tabular}

Table 2. Activities of daily living among older Saudi adults

\begin{tabular}{lcc}
\hline Activity of Daily Living (ADL) & $\begin{array}{c}\text { Frequency } \\
(\mathrm{N}=55)\end{array}$ & $\%$ \\
\hline Self-reliant & 14 & $25.4 \%$ \\
Require little help & 20 & $36.4 \%$ \\
Require a lot of help & 12 & $22 \%$ \\
Fully dependent & 9 & $16.3 \%$ \\
\hline
\end{tabular}

Table 3. Activity level among older Saudi adults

\begin{tabular}{lcc}
\hline Activity level & $\begin{array}{c}\text { Frequency } \\
(\mathrm{N}=55)\end{array}$ & $\%$ \\
\hline Sedentary level & 38 & $69.0 \%$ \\
One hour weekly activity & 9 & $16.4 \%$ \\
More than two hours weekly activity & 8 & $14.65 \%$ \\
\hline
\end{tabular}

Table 4. Sleep difficulty among older Saudi adults

\begin{tabular}{ccc}
\hline Sleep difficulty & $\begin{array}{c}\text { Frequency } \\
(\mathrm{N}=55)\end{array}$ & $\%$ \\
\hline Yes & 39 & $70 \%$ \\
No & 16 & $29 \%$ \\
\hline
\end{tabular}

Table 5. Causes of falls among older Saudi adults

\begin{tabular}{lcc}
\hline $\begin{array}{l}\text { Cause of falls among } \\
\text { the elderly }\end{array}$ & $\begin{array}{c}\text { Frequency } \\
(\mathrm{N}=55)\end{array}$ & $\%$ \\
\hline Environment & 29 & $74.3 \%$ \\
Dizziness & 12 & $21.8 \%$ \\
Gait disorders & 11 & $20 \%$ \\
Drop attack & 2 & $3.6 \%$ \\
Syncope & 1 & $1.8 \%$ \\
\hline
\end{tabular}

\section{DISCUSSION}

Health status is an important factor that significantly impacts the QoL of the elderly population. The major elements of health status are perceived health, especially psychological well-being, chronic illnesses, and functional status (e.g., fall risk). Some factors that influence health-related QoL are institutionalization, reduced life expectancy, cognitive impairment, disability, chronic pain, social isolation, and functional status. In the current study, researchers found sleep disorders and chronic diseases were associated with an increased risk of falls, leading to a poorer QoL among older adults. Fall injuries are the greatest threat to the quality of health and well-being of older adults ${ }^{8)}$. However, this study found that $51 \%(\mathrm{n}=29)$ of participants had fallen once and $49 \%(n=27)$ had fallen more than once in the past 12 months. Furthermore, this study indicated a high positive correlation between age and fall history $(r=0.06)$. Similarly, another study has reported that people aged 85 years and older have a greater risk of falls or fall-related injuries compared to those in the 65-85 age range ${ }^{9)}$. More detailed questions about sleeping disorders revealed that participants experienced difficulty sleeping (65.22\%), difficulty falling back to sleep after waking (63.04\%), feeling drowsy when awake $(41.30 \%)$, and feeling exhausted during the day as the result of sometimes $(50 \%)$ or often $(17.39 \%)$ having problems sleeping at night. In addition, difficulty falling asleep varied between sometimes $(36.96 \%)$ and often $(32.61 \%)$. Thus, sleep disturbances are often experienced by older adults ${ }^{9-11)}$ and were prevalent in older adults (65.2\%). This study also found a clear relationship between sleeping difficulties and falls. Similar results from a study of 150 participants reported that falls and sleep disturbances were common during the previous year, as evident in the presence of participants' reporting falls (44\% vs. $41 \%)$ and multiple falls (20\% vs. $16 \%)$. In contrast, one study shows that more than a third of community-dwelling women $(37.7 \%)$ reported at least one night-time sleep problem, and $8.1 \%$ reported abnormal daytime sleepiness ${ }^{12}$. Abnormal daytime sleepiness was also significantly prevalent among those who experienced falls but no association has been found between night-time sleep problems and increased fall risk $^{9}$. Additionally, the present study shows that the older adults with chronic diseases who had a higher risk of falls $(43.5 \%)$ had more than two chronic conditions, such as DM (58.18\%) and HTN (29.0\%). Previous studies have confirmed that older adults with chronic diseases have a greater risk of falling. The percentage of older adults with two or more chronic conditions increased for men and women; $45 \%$ of older adults aged 65 and over had been diagnosed with two or more chronic diseases. Another study revealed that older women with any chronic diseases had a higher risk of falling (32\%) than those who used psychotropic drugs (2-5\%). Accordingly, it suggested preventing falls using strategies focusing on prevention and control of chronic disease should be used rather than polypharmacy ${ }^{13)}$. Consequently, there is widespread agreement that older adults' poor sleep habits affect QoL. Likewise, another study confirmed that elderly people without any physical disease enjoyed better sleep quality and QoL than those with more than two physical diseases, particularly 
those suffering from heart disease ${ }^{14)}$. Thus, health professionals should consider sleep disorders and chronic diseases risk factors for a poor QoL among older people.

Further research is strongly needed to investigate the impact of specific chronic diseases, such as DM, on the risk of falling in the Saudi community. Additionally, the present study reports that poor vision and hearing affected $40.09 \%$ of older adults, which may subsequently influence QoL. Similarly, previous research has confirmed that poor QoL was more frequent in older people with a combination of poor vision and other sensory and balance impairments. Specifically, older people with vision and hearing loss were more likely to have fallen in the past year $(37.6 \%)$ than those without vision or hearing impairment (16.5\%). In addition, using mobility aids and assistive devices increase older adults' confidence and sense of security, which promotes increased activity level and independence ${ }^{15,16}$. The relation between using an assistive device and the risk of falling remains unclear ${ }^{17)}$. For instance, a prospective prediction of increased fall risk among older adults using mobility aids has been found as the result of disrupted balance control when using an aid without sufficient training or lack of necessary skills. In this study, $39.13 \%$ of participants reported walking with mobility aids, indicating a correlation between mobility aid use and increased fall risk that can affect QoL. Nonetheless, this study confirms that older Saudi adults engage in little physical activity (69\%), which can predispose them to a risk of developing a disability or a chronic disease such as DM. This will adversely affect their mobility and reduce ability to perform ADL. Thus, the greater number of risk factors an individual has, the more likely they are to fall and the more serious the results of falls will be ${ }^{18)}$. Furthermore, this study reports that single sensory impairments (either visual or auditory) are significantly and independently linked with a high risk of depression and reduced ADL self-sufficiency. Thus, the QoL of elderly people is significantly linked to sensory impairment. Mood levels and social relationships are particularly influenced by visual impairment, whereas self-sufficiency in daily living is more strongly related to hearing impairment ${ }^{19,20)}$. However, the Kingdom remains in the early stages of development, despite that chronic diseases are now the primary conditions for which older people seek health care. However, since there are no special hospital wards for older patients in Saudi Arabia, they are treated by general internists, highlighting the need for a costeffective national program for older Saudi people ${ }^{21-23)}$. To shed additional light on these issues, a Saudi Arabian study was conducted by the Department of Family and Community Medicine to elicit decision makers' (both medical and nonmedical) opinions on the types of facilities, their locations, and culturally acceptable levels of service for the health care of the older population ${ }^{24)}$. The conclusions from this study were that the majority of respondents favored the establishment of special health care facilities that are able to directly respond to older peoples' health needs. We recommend promoting healthy lifestyles and behavioral changes through a community-based health education program. Screening programs for chronic diseases among the elderly population (especially for DM and HTN) are also highly recommended. Additionally, there is a need for satisfactory living conditions for the elderly; this would satisfy their psychosocial needs, increase ADL, and increase participation in useful activities. Moreover, welfare services and heath planners should be encouraged to provide good quality training for elderly home care services, similar to those in Western countries. These should be culturally and religiously acceptable, and include residential accommodation, sheltered housing, and rest homes for older people.

\section{ACKNOWLEDGEMENTS}

This project was funded by the Deanship of Scientific Research (DSR), King Abdulaziz University, Jeddah, Saudi Arabia under grant No. (142-004-D1434).The authors, therefore, acknowledge with thanks DSR technical and financial support.

\section{REFERENCES}

1) World Health Organization: Towards policy for health \& ageing, 2010.

2) Fielding P: Attitudes Revisited: An examination of student nurses' attitudes towards old people in hospital. Tonbridge: Whitefriars' Press, 1986.

3) Abalkheil R: The development of a model for a sociomedical facility for elderly people in Arabia (PhD Dissertation). Boston University, 1988.

4) Al-Sadhan AN: Elderly and family social characteristics in Arabia. In: Ministry of Work and Social Welfare (Ed.), Riyadh: Ministry of Work and Social Welfare Press, 2000.

5) Mahfouz AA, Al-Sharif AI, El-Gama MN, et al.: Primary health care services utilization and satisfaction among the elderly in Asir region, Saudi Arabia. East Mediterr Health J, 2004, 10: 365-371. [Medline]

6) Mufti MH: A case for community and hospital-based long-term care facilities in Saudi Arabia. Ann Saudi Med, 2002, 22: 336-338. [Medline]

7) Al-Senany S: Risk Management in Hospital and Prevention of Patient Accidents. MSc dissertation, University Of Wales, Swansea, United Kingdom, 2003.

8) Alsenany S: Prevention of patient falls in University Hospital. Generation review. Br Soc Gerontol J, 2010, 21: 1-5.

9) Grundstrom AC, Guse CE, Layde PM: Risk factors for falls and fall-related injuries in adults 85 years of age and older. Arch Gerontol Geriatr, 2012, 54: 421-428. [Medline] [CrossRef]

10) Helbig AK, Döring A, Heier M, et al.: Association between sleep disturbances and falls among the elderly: results from the German Cooperative Health Research in the Region of Augsburg-Age study. Sleep Med, 2013, 14: 1356-1363. [Medline] [CrossRef]

11) Rubenstein LZ: Falls in older people: epidemiology, risk factors and strategies for prevention. Age Ageing, 2006, 35: ii37-ii41. [Medline] [CrossRef]

12) Stone KL, Ensrud KE, Ancoli-Israel S: Sleep, insomnia and falls in elderly patients. Sleep Med, 2008, 9: S18-S22. [Medline] [CrossRef]

13) Reid KJ, Baron KG, Lu B, et al.: Aerobic exercise improves self-reported sleep and quality of life in older adults with insomnia. Sleep Med, 2010, 11: 934-940. [Medline] [CrossRef]

14) Paudel ML, Taylor BC, Diem SJ, et al. Osteoporotic Fractures in Men Study Group: Association between depressive symptoms and sleep disturbances in community-dwelling older men. J Am Geriatr Soc, 2008, 56: 1228-1235. [Medline] [CrossRef]

15) Sattin RW: Falls among older persons: a public health perspective. Annu Rev Public Health, 1992, 13: 489-508. [Medline] [CrossRef]

16) Berg KO, Maki BE, Williams JI, et al.: Clinical and laboratory measures of postural balance in an elderly population. Arch Phys Med Rehabil, 1992, 73: 1073-1080. [Medline]

17) Asakawa $Y$, Ikezoe $T$, Hazaki $K$, et al.: Relationship between falls and knee extension strength in the elderly. J Phys Ther Sci, 1996, 8: 45-48. [CrossRef]

18) Yu W, An C, Kang H: Effects of resistance exercise using thera-band on balance of elderly adults: a randomized controlled trial. J Phys Ther Sci, 2013, 25: 1471-1473. [Medline] [CrossRef]

19) Lee $\mathrm{CW}$, Cho GH: Effect of stationary cycle exercise on gait and balance of elderly women. J Phys Ther Sci, 2014, 26: 431-433. [Medline] [CrossRef]

20) Kang KY: Effects of visual biofeedback training for fall prevention in the elderly. J Phys Ther Sci, 2013, 25: 1393-1395. [Medline] [CrossRef] 
21) Al-Shammari SA, Al Mazrou Y, Jarallah JS, et al.: Appraisal of clinical, psychosocial, and environmental health of elderly in Saudi Arabia a household survey. Int J Aging Hum Dev, 2000, 50: 43-60. [Medline] [CrossRef]

22) Al-Shammari SA: Causes of ill health among elderly patients seen at a primary care clinic at King Khaild university Hospital, Arabia. Med J, 1994, 15: 358-363.

23) al-Shammari SA, Felemban FM, Jarallah JS, et al.: Culturally acceptable health care services for Saudi's elderly population: the decision-maker's perception. Int J Health Plann Manage, 1995, 10: 129-138. [Medline] [CrossRef]

24) al-Shammari S, Jarallah J, Felimban F, et al.: Clinical and epidemiological pattern of long-stay in-patients: an opportunity for the provision of outreach community services in Saudi Arabia. J Public Health Med, 1995, 17: 179-186. [Medline] 